\title{
Fluid Flow in a Pipe
}

\author{
U Sujatha $^{1}$, C Sarada ${ }^{2}$
}

\begin{abstract}
We study the fluid flow through a network of intersected thin pipes with prescribed pressure at their ends. Pipes are either thin or long and the ratio between the length and the cross-section is considered as the small parameter. Using the asymptotic analysis with respect to that small parameter the effective behavior of the flow is found. At each junction an explicit formula for computing the value of the pressure is found. The interior layer phenomenon in vicinity of the junction is studied. We generalize the junction formula on the case of adiabatic compressible flow.
\end{abstract}

Keywords: Fluid flow, pipes network, pressure value at junction

\section{Introduction}

In everyday life, we recognize three states of matter: solid, liquid and gas. Although different in many respects, liquids and gases have a common characteristic in which they differ from solids: they are fluids, lacking the ability of solids to offer a permanent resistance to a deforming force.

A fluid is a substance which deforms continuously under the action of shearing forces, however small they may be. Conversely, it follows that: If a fluid is at rest, there can be no shearing forces acting and, therefore, all forces in the fluid must be perpendicular to the planes upon which they act.

\section{Fluid Flow in Pipes}

We will be looking here at the flow of real fluid in pipes real meaning a fluid that possesses viscosity hence looses energy due to friction as fluid particles interact with one another and wall. Recall from Level 1 that the pipe the shear stress induced in a fluid flowing near a boundary is given by Newton's law of viscosity:

$$
\tau \propto \mathrm{du} / \mathrm{dy}
$$

This tells us that the shear stress, $\tau$, in a fluid is proportional to the velocity gradient - the rate of change of velocity across the fluid path. For a "Newtonian" fluid we can write

$$
\tau \mu=\mathrm{du} / \mathrm{dy}
$$

Where the constant of proportionality, $\mu$, is known as the coefficient of viscosity (or simply viscosity). Recall also that flow can be classified into one of two types, laminar or turbulent flow (with a small transitional region between these two). The non-dimensional number, the Reynolds number, $\mathrm{Re}$, is used

$$
\operatorname{Re}=\rho u d / \mu
$$

\section{For A Flow Pipe}

Laminar flow: $\operatorname{Re}<2000$

Transitional flow: $2000<\operatorname{Re}<4000$

Turbulent flow: $\operatorname{Re}>4000$

It is important to determine the flow type as this governs how the amount of energy lost to friction relates to the velocity of the flow. And hence how much energy must be used to

\section{Move The Fluid}

Pressure loss due to friction in a pipeline. Consider a cylindrical element of incompressible fluid flowing in the pipe, as shown

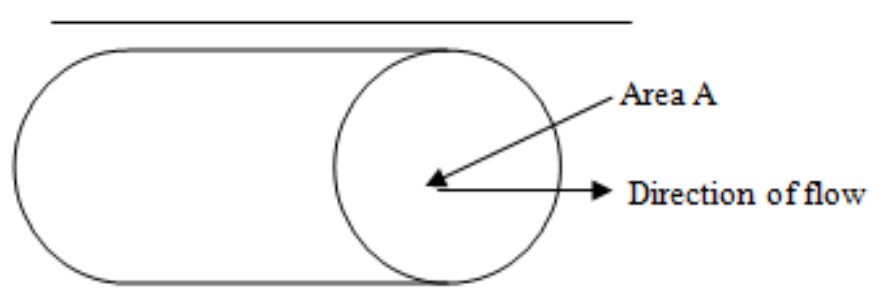

Figure 1: Element of fluid in a pipe

The pressure at the upstream end, 1 , is $\mathrm{p}$, and at the downstream end, 2 , the pressure has fallen by $\Delta p$ to (p- $\Delta p)$.

The driving force due to pressure $(\mathrm{F}=$ Pressure $\mathrm{x}$ Area $)$ can then be written driving force $=$ Pressure force at 1 - pressure force at 2

$$
\mathrm{pA}-(\mathrm{p}-\Delta \mathrm{p}) \mathrm{A}=\Delta \mathrm{pA}=\Delta \mathrm{p} \frac{\mu d^{2}}{4}
$$

The retarding force is that due to the shear stress by the walls

$=$ shear stress $\mathrm{x}$ area over which it acts

$=\tau_{W} \mathrm{x}$ area of pipe wall

$=\tau_{\mathrm{w}} \pi \mathrm{dL}$

As the flow is in equilibrium,

Driving force $=$ retarding force

$$
\begin{aligned}
\Delta \mathrm{p} \frac{\mu d^{2}}{4} & =\tau_{\mathrm{w}} \pi \mathrm{dL} \\
\Delta \mathrm{p} & =\frac{\tau_{w} A L}{d}
\end{aligned}
$$

Giving an expression for pressure loss in a pipe in terms of the pipe diameter and the shear stress at the wall on the pipe. The shear stress will vary with velocity of flow and hence with Re. 


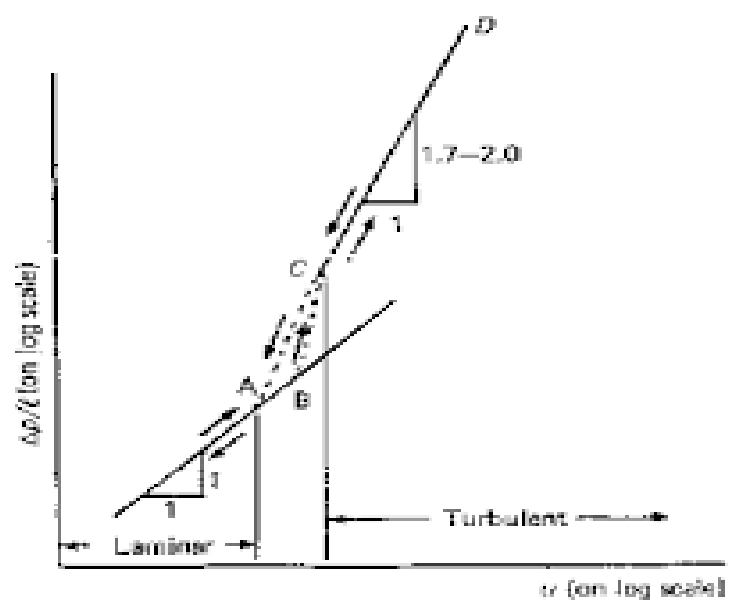

\section{Relationship between Velocity and Pressure Loss in Pipes}

This graph shows that the relationship between pressure loss and $\mathrm{Re}$ can be expressed as

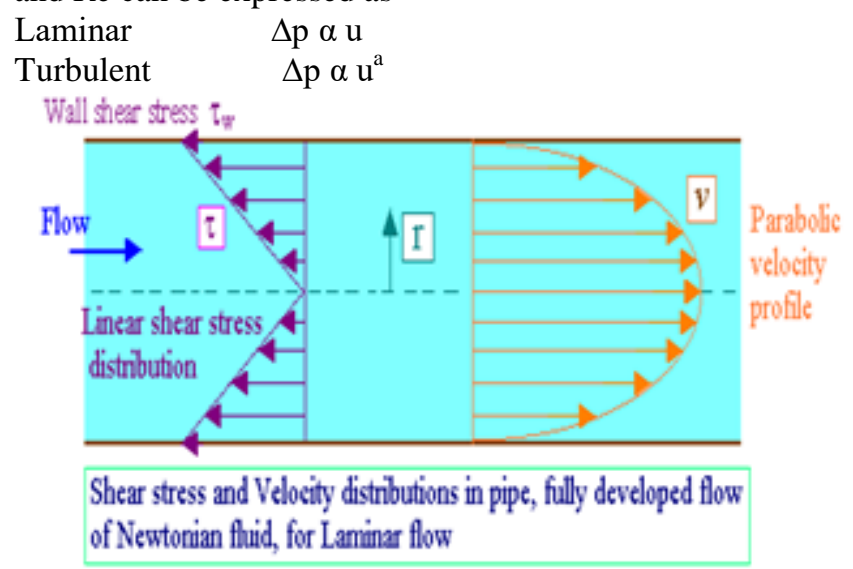

\section{Turbulent Flow}

The head loss in turbulent flow in a circular pipe is given by, $\mathrm{h}_{\mathrm{f}}=2 \mathrm{fLv}^{2} / \mathrm{D}=\Delta \mathrm{p} / \rho$

where $\mathrm{f}$ is the friction factor, defined as $\mathrm{f}=\tau_{\mathrm{w}} /\left(\rho \mathrm{v}^{2} / 2\right)$

where $\tau_{\mathrm{w}}$ is wall shear stress.

The value of friction factor $f$ depends on the factors such as velocity (v), pipe diameter (D), density of fluid $(\rho)$, viscosity of fluid $(\mu)$ and absolute roughness $(\mathrm{k})$ of the pipe.

These variables are grouped as the dimensional numbers NRe and $\mathrm{k} / \mathrm{D}$

Where $\mathrm{NRe}=\mathrm{Dv} \rho / \mu=$ Reynolds number and $\mathrm{k} / \mathrm{D}$ is the relative roughness of the pipe.

Blasisus, in 1913 was, the first to propose an accurate empirical relation for the friction factor in turbulent flow in smooth pipes, namely

$$
\mathrm{f}=0.079 / \mathrm{NRe}^{0.25}
$$

This expression yields results for head loss to \pm 5 percent for smooth pipes at Reynolds numbers up to 100000 .

For rough pipes, Nikuradse, in 1933, proved the validity of $\mathrm{f}$ dependence on the relative roughness ratio $\mathrm{k} / \mathrm{D}$ by investigating the head loss in a number of pipes which had been treated internally with a coating of sand particles whose size could be varied.

Thus, the calculation of losses in turbulent pipe flow is dependent on the use of empirical results and the most common reference source is the Moody chart, which is a logarithmic plot of $\mathrm{f}$ vs. NRe for a range of $\mathrm{k} / \mathrm{D}$ values. A typical Moody chart is presented as figure.

There are a number of distinct regions in the chart.

1) The straight line labeled 'laminar flow', representing $\mathrm{f}=$ $16 / \mathrm{NRe}$, is a graphical representation of the Poiseuille equation. The above equation plots as a straight line of slope -1 on a log-log plot and is independent of the pipe surface roughness.

2) For values of $k / D<0.001$ the rough pipe curves approach the Blasius smooth pipe curve.

\section{Conclusion}

An attempt has been made in this experimental apparatus to study pressure losses in various components in a piping system consisting of straight pipes, sudden expansion and contraction, bends and valves. The general trends and magnitudes obtained during experiments will be the indication of the pressure loss from various components in the pipe system. However, in practical situations, such combinations are not adopted over a short span length. The normally accepted design criterion for pressure tapping's is 30 to 50 pipe diameters away from obstruction. Also, sufficient pipe length has been left between each component in the circuit to eliminate any adverse influence of the neighboring components. Thus, any discrepancies between experimental and theoretical results may be attributed due to three main factors;

\section{References}

[1] F. M. White, 1999. Fluid Mechanics, McGraw-Hill.

[2] B. R. Munson, D.F Young and T. H. Okiisshi, 1998. Fundamentals of Fluid Mechanics, John Wiley and Sons, Inc.

[3] Y. Nakayama and R.F. Boucher, 1999.Intoduction to Fluid Mechanics, Butterworth Heinemann.

[4] F.M. White, Fluid Mechanics, 7th Edition, McGraw-Hill, New York, 2011 


\section{International Journal of Science and Research (IJSR)}

ISSN (Online): 2319-7064

Index Copernicus Value (2015): 78.96 | Impact Factor (2015): 6.391

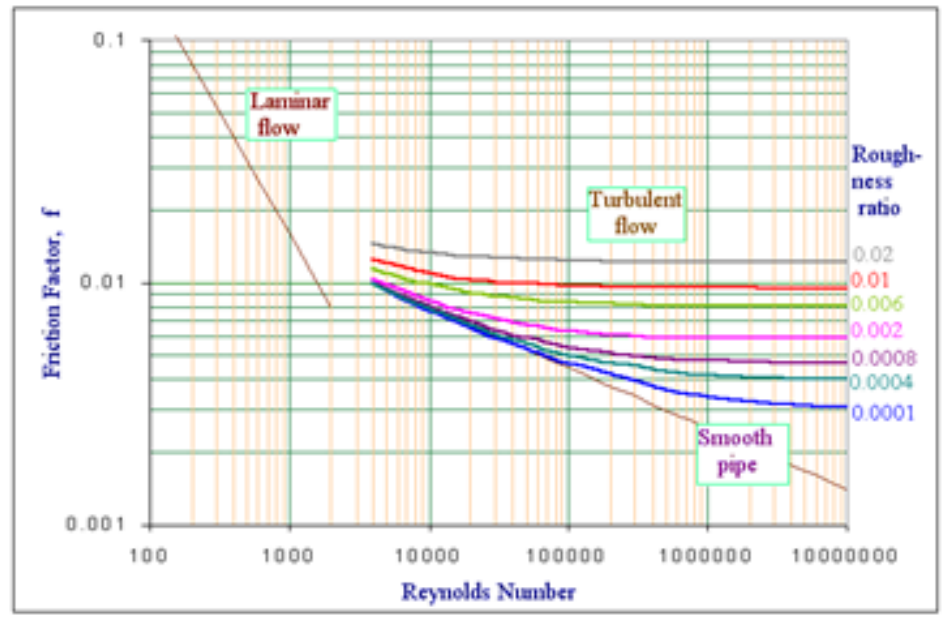

Fanuing Friction Factor for Flow in Pipes.

Volume 6 Issue 7, July 2017 www.ijsr.net 